Supplement of Biogeosciences, 15, 4245-4269, 2018 https://doi.org/10.5194/bg-15-4245-2018-supplement (c) Author(s) 2018. This work is distributed under the Creative Commons Attribution 4.0 License.

(c) (1)

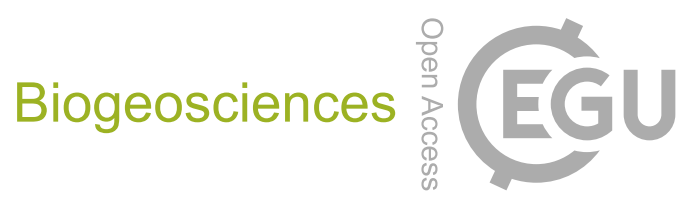

Supplement of

\title{
Large but decreasing effect of ozone on the European carbon sink
}

Rebecca J. Oliver et al.

Correspondence to: Rebecca Oliver (rfu@ceh.ac.uk)

The copyright of individual parts of the supplement might differ from the CC BY 4.0 License. 


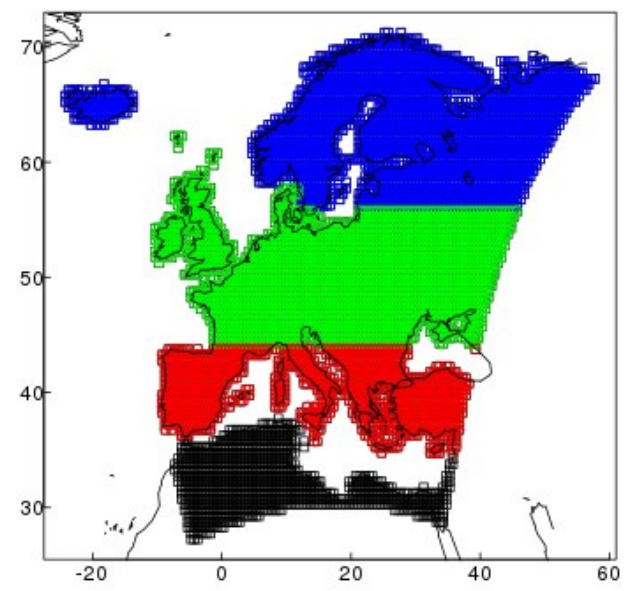

4 Figure S1. Regions of model domain, blue is Boreal, green is Temperate, red is Mediterranean.

5

6
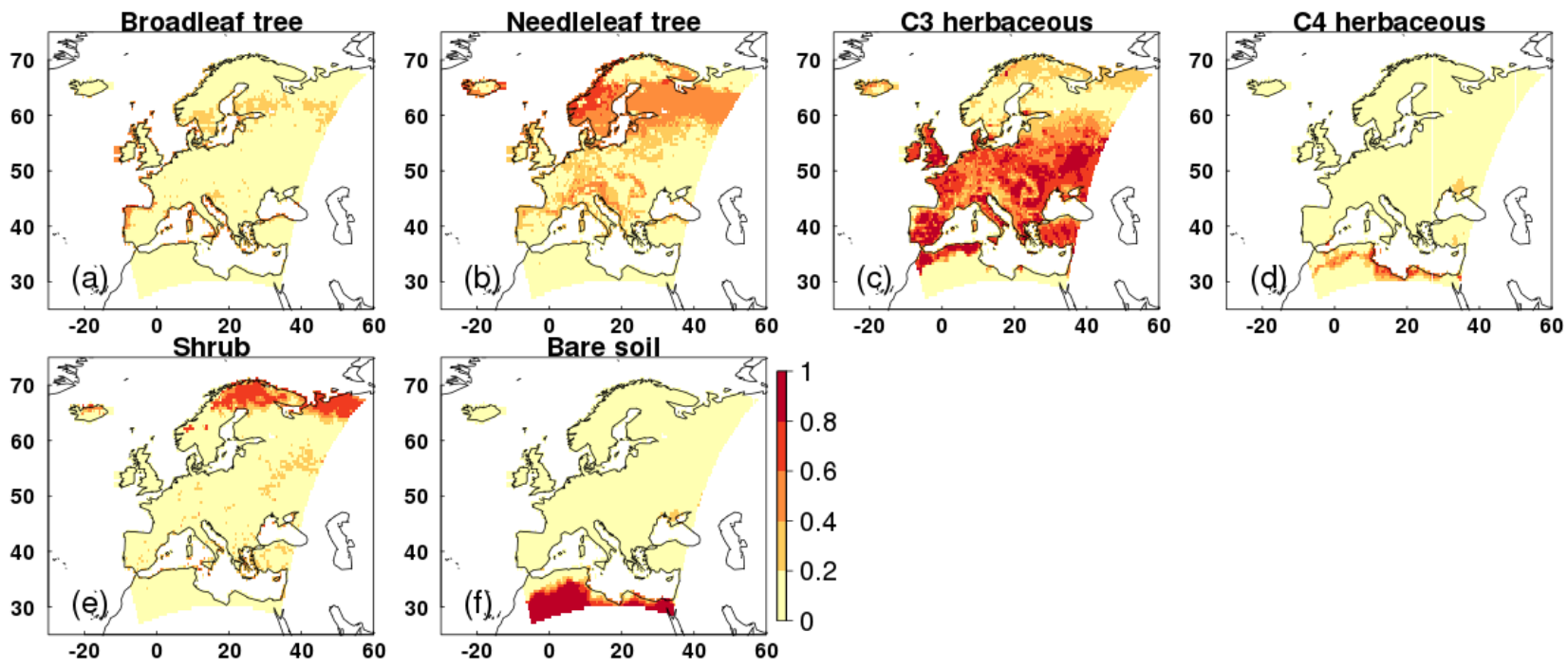

Figure S2. Fractional cover of each JULES PFT and bare soil at $0.5^{\circ} \times 0.5^{\circ}$ resolution. 

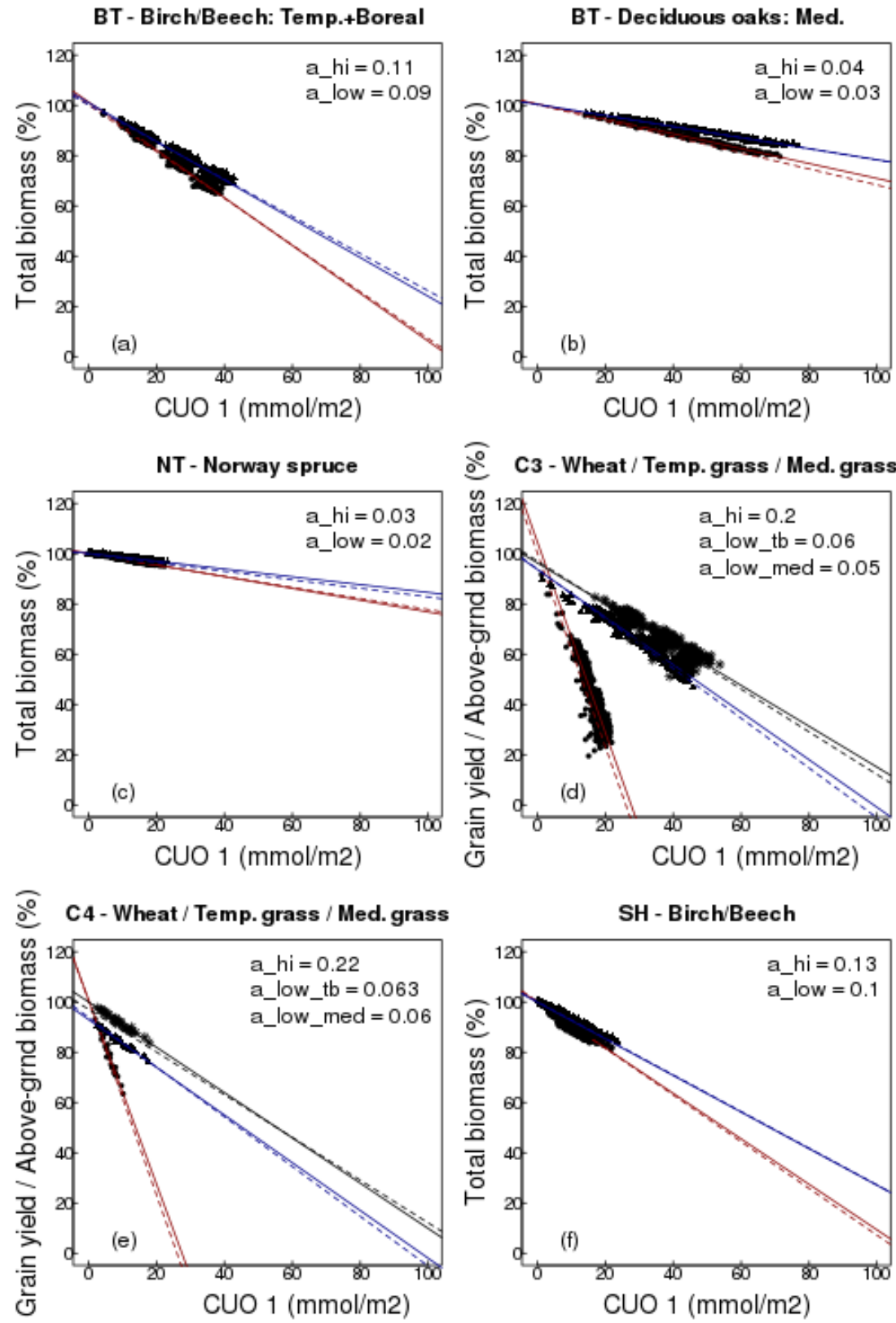

Figure S3. Calibration of JULES for $\mathrm{O}_{3}$ impacts on plant productivity for each JULES PFT ; a) broadleaf tree - temperate/boreal, b) broadleaf tree Mediterranean, c) Needle leaf tree, d) $\mathrm{C}_{3}$ herbaceous (split into temperate/boreal and Mediterranean for the natural grasslands), e) $\mathrm{C}_{4}$ herbaceous (split into temperate/boreal and Mediterranean for the natural grasslands), and f) shrub. High (red) and low (blue) plant $\mathrm{O}_{3}$ sensitivities are shown. For the herbaceous PFTs the low sensitivity calibration is separate for Mediterranean regions (black). The solid line is the regression line through the modelled points, the dashed line is the regression line from the observed dose-response relationship. The $\mathrm{x}$ axis is cumulative uptake of $\mathrm{O}_{3}(\mathrm{CUO})$ above the critical $\mathrm{O}_{3}$ threshold $\left(F_{\text {O3crit }}\right)$. 


\begin{tabular}{|c|c|c|c|c|c|}
\hline & \multicolumn{5}{|c|}{ High Sensitivity } \\
\hline & BT & NT & $\mathrm{C3}$ & C4 & SH \\
\hline$F_{O 3 c r i t}\left(\mathrm{nmol} / \mathrm{m}^{2} / \mathrm{s}\right)$ & 1.00 & 1.00 & 1.00 & 1.00 & 1.00 \\
\hline$a\left(\mathrm{mmol} / \mathrm{m}^{2}\right)$ & 0.110 & 0.030 & 0.200 & 0.220 & 0.130 \\
\hline Function & $\begin{array}{l}\text { Birch/Beech: } \\
\mathrm{y}=100.2-0.93 \mathrm{x}\end{array}$ & $\begin{array}{c}\text { Norway spruce: } \\
y=99.8-0.22 x\end{array}$ & $\begin{array}{c}\text { Wheat: } \\
y=100.3-3.85 x\end{array}$ & $\begin{array}{c}\text { Wheat: } \\
y=100.3-3.85 x\end{array}$ & $\begin{array}{c}\text { Birch/Beech: } \\
y=100.2-0.93 x\end{array}$ \\
\hline dqcrit $\left(\mathrm{kg} \mathrm{kg}^{-1}\right)$ & 0.09 & 0.06 & 0.1 & 0.075 & 0.1 \\
\hline fo & 0.875 & 0.875 & 0.9 & 0.8 & 0.9 \\
\hline \multirow[t]{3}{*}{$g_{1}\left(k P a^{0.5}\right)$} & 3.22 & 2.22 & 5.56 & 1.1 & 2.24 \\
\hline & \multicolumn{5}{|c|}{ Low Sensitivity } \\
\hline & BT & NT & C3 & $\mathrm{C4}$ & SH \\
\hline Fo3crit $\left(\mathrm{nmol} / \mathrm{m}^{2} / \mathrm{s}\right)$ & 1.00 & 1.00 & 1.00 & 1.00 & 1.00 \\
\hline$a\left(\mathrm{mmol} / \mathrm{m}^{2}\right)$ & 0.090 & 0.020 & 0.060 & 0.063 & 0.100 \\
\hline \multirow[t]{3}{*}{ Function } & $\begin{array}{c}\text { Birch/Beech: } \\
\mathrm{y}=100.2-0.74 \mathrm{x}\end{array}$ & $\begin{array}{c}\text { Norway spruce: } \\
y=99.8-0.17 x\end{array}$ & $\begin{array}{c}\text { Temperate } \\
\text { perennial } \\
\text { grassland: } \\
\mathrm{y}=93.9-0.99 \mathrm{x} \\
\end{array}$ & $\begin{array}{c}\text { Temperate } \\
\text { perennial } \\
\text { grassland: } \\
\mathrm{y}=93.9-0.99 \mathrm{x}\end{array}$ & $\begin{array}{c}\text { Birch/Beech: } \\
y=100.2-0.74 x\end{array}$ \\
\hline & \multicolumn{5}{|c|}{ High Sensitivity } \\
\hline & BT - Med. & & & & \\
\hline$F_{O 3 c r i t}\left(\mathrm{nmol} / \mathrm{m}^{2} / \mathrm{s}\right)$ & 1.00 & & & & \\
\hline$a\left(\mathrm{mmol} / \mathrm{m}^{2}\right)$ & 0.040 & & & & \\
\hline \multirow[t]{3}{*}{ Function } & $\begin{array}{c}\text { Dec. Oaks: } \\
\mathrm{y}=100.3-0.32 \mathrm{x}\end{array}$ & & & & \\
\hline & \multicolumn{5}{|c|}{ Low Sensitivity } \\
\hline & BT - Med. & C3 - Med. & C4 - Med. & & \\
\hline$F_{O 3 c r i t}\left(\mathrm{nmol} / \mathrm{m}^{2} / \mathrm{s}\right)$ & 1.00 & 1.00 & 1.00 & & \\
\hline$a\left(\mathrm{mmol} / \mathrm{m}^{2}\right)$ & 0.030 & 0.050 & 0.060 & & \\
\hline Function & $\begin{array}{c}\text { Dec. Oaks: } \\
y=100.3-0.22 x\end{array}$ & $\begin{array}{c}\text { Mediterranean } \\
\text { annual pasture: } \\
y=97.1-0.85 x\end{array}$ & $\begin{array}{c}\text { Mediterranean } \\
\text { annual pasture: } \\
y=97.1-0.85 x\end{array}$ & & \\
\hline
\end{tabular}

Table S1. PFT-specific parameter values used in the $\mathrm{O}_{3}$ uptake and $g_{s}$ formulation in JULES. $F_{O 3 c r i t}$ is the critical $\mathrm{O}_{3}$ threshold above which damage occurs, $a$ determines the reduction in photosynthesis with $\mathrm{O}_{3}$ exposure, 'function' shows the regression equation for the observed functions ( $\mathrm{x}$ is $\left.F_{O 3 c r i t}\right), d q_{\text {crit }}\left(\mathrm{kg} \mathrm{kg}^{-1}\right)$ is a PFT specific parameters representing the critical humidity deficit at the leaf surface (used in the default JULES $g_{s}$ model), $f_{0}$ is the leaf internal to atmospheric $\mathrm{CO}_{2}$ ratio $\left(c_{i} / c_{a}\right)$ at the leaf specific humidity deficit (also used in the default JULES $g_{s}$ model), and $g_{l}$ is the PFT specific parameter of the Medlyn et al., (2011) $g_{s}$ model. The parameters $d q_{c r i t}, f_{0}$ and $g_{l}$ vary by PFT, but not by $\mathrm{O}_{3}$ sensitivity so are only shown once here. 


\begin{tabular}{|c|c|c|c|c|c|c|}
\hline Site name & Country & Latitude & Longitude & $\begin{array}{l}\text { Simulated } \\
\text { years }\end{array}$ & Land cover & Dominant PFT(s) \\
\hline US-UMB & USA & 45.56 & -84.71 & $2000-2014$ & Broadleaf forest & $100 \% \mathrm{BT}$ \\
\hline IT-CA1 & Italy & 42.38 & 12.02 & $2011-2014$ & Broadleaf forest & $100 \% \mathrm{BT}$ \\
\hline FI-Hyy & Finland & 62 & 24.3 & $1996-2014$ & Needleleaf forest & $100 \% \mathrm{NT}$ \\
\hline DE-Tha & Germany & 51 & 13.57 & $1996-2014$ & Needleleaf forest & $100 \% \mathrm{NT}$ \\
\hline CH_Cha & Switzerland & 47.21 & 8.41 & $2006-2014$ & C3 grassland & $80 \% \mathrm{C} 3,20 \%$ bare soil \\
\hline US-SRG & USA & 31.8 & -110.83 & $2008-2014$ & C4 grassland & $\begin{array}{l}80 \% \text { C4, } 20 \% \text { bare soil } \\
50 \% \text { BT, } 15 \% \text { C4, } 25 \% \text { shrub, } 10 \% \text { bare }\end{array}$ \\
\hline CG-Tch & Congo & -4.5 & 11.66 & 2006-2009 & Deciduous savanna & soil \\
\hline
\end{tabular}

Table S2. Sites from the FLUXNET2015 dataset used in the site simulations to evaluate $g s$ models.
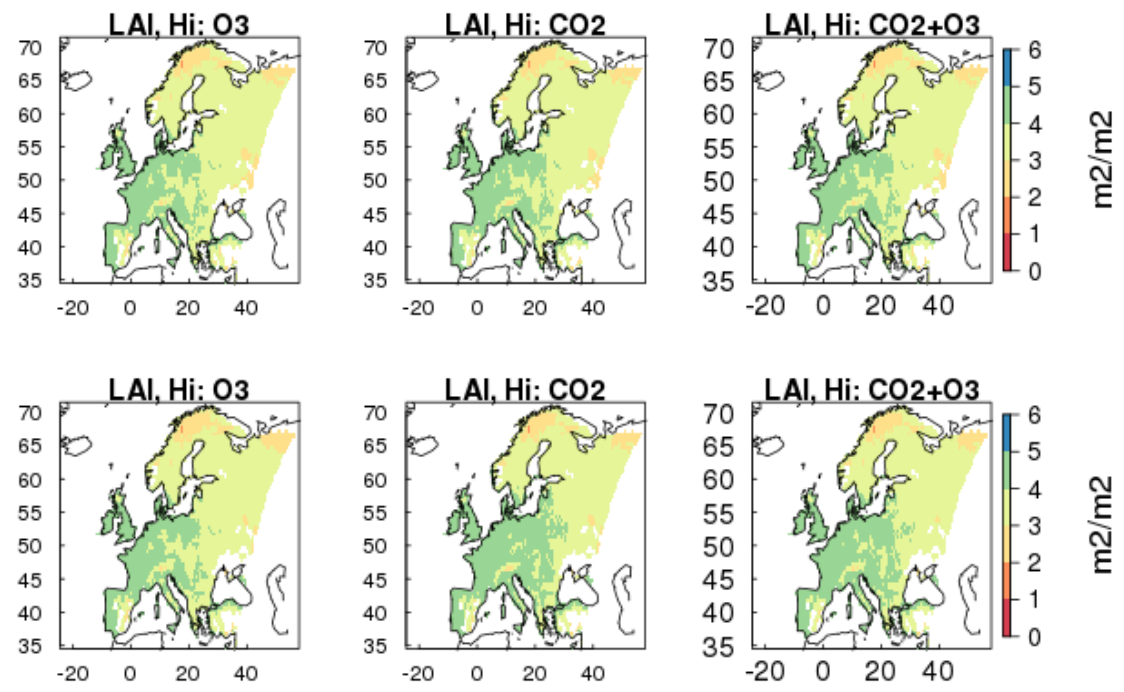

है
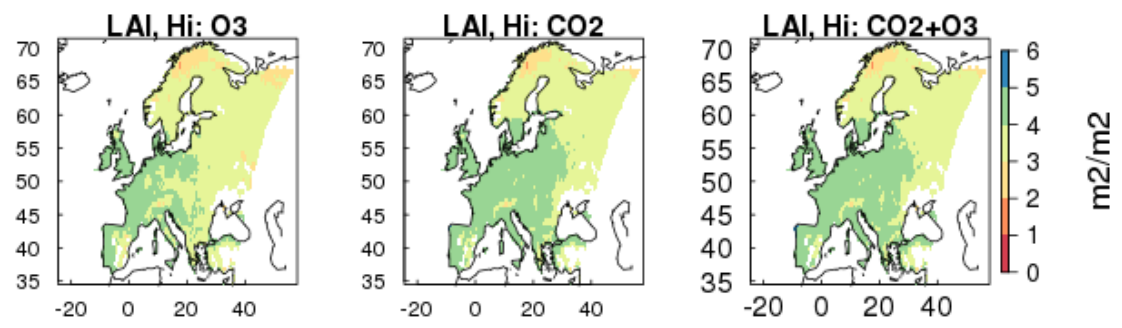

Figure. S4. Simulated mean annual leaf area index (LAI) in1901 (top row), 2001 (middle row) and 2050 (bottom row) for the 44 high plant $\mathrm{O}_{3}$ sensitivity. 

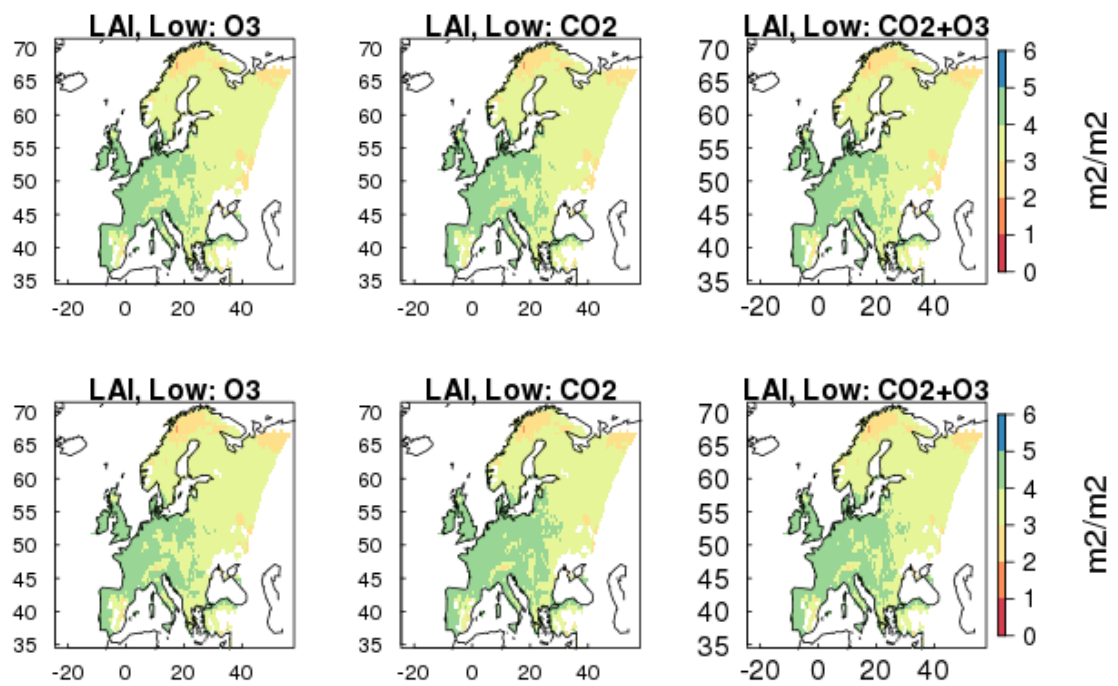

\section{กิ}
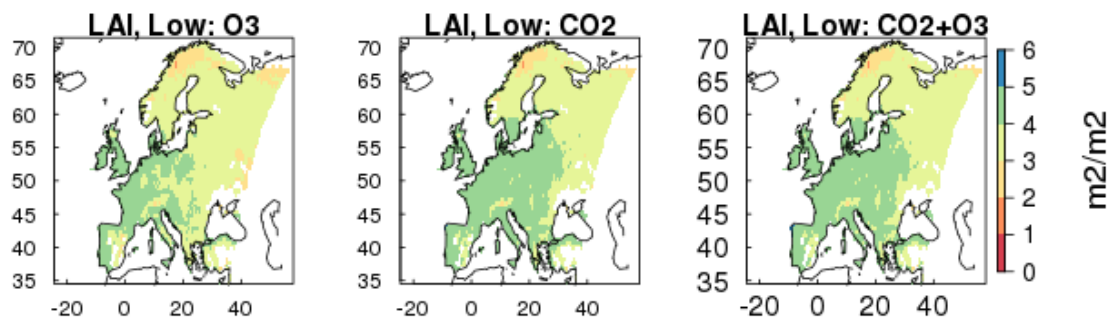

Figure. S5. Simulated mean annual leaf area index (LAI) in1901 (top row), 2001 (middle row) and 2050 (bottom row) for the low plant $\mathrm{O}_{3}$ sensitivity.
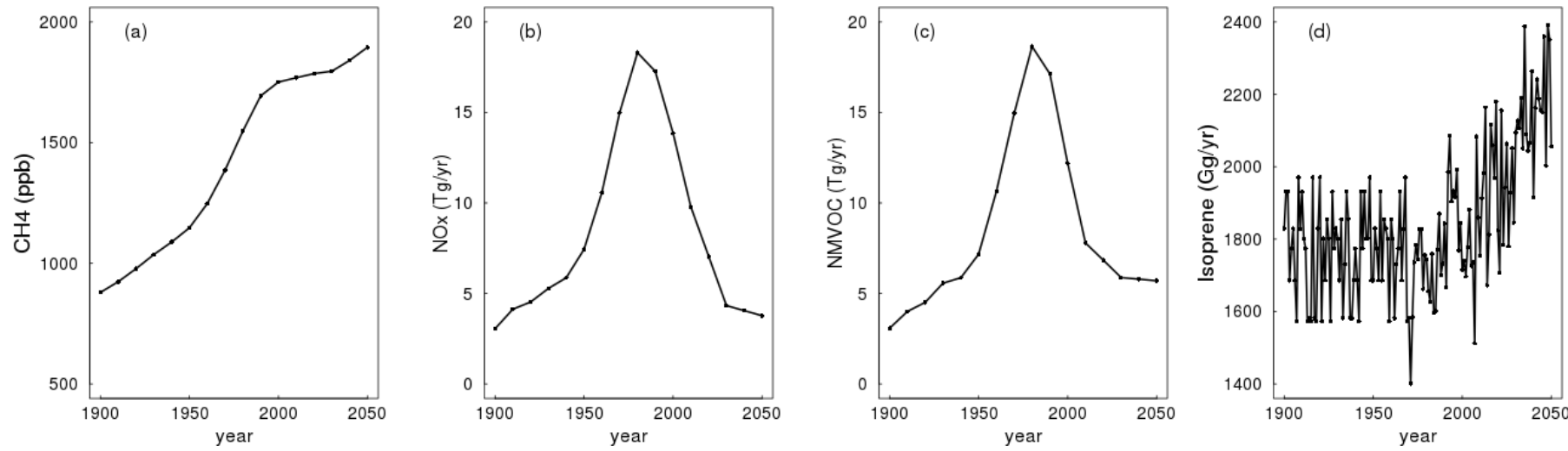

Figure. S6. Trend in the emissions of the $\mathrm{O}_{3}$ precursors a) methane $\left(\mathrm{CH}_{4}(\mathrm{ppb})\right)$, b) nitrous oxides $\left(\mathrm{NO}_{\mathrm{x}}(\mathrm{Tg} / \mathrm{yr})\right)$, c) non-methane VOC (NMVOC ( $\mathrm{Tg} / \mathrm{yr})$ ) and d) Isoprene ( $\mathrm{Gg} / \mathrm{yr}$ ) over Europe from 1900 to 2050. 

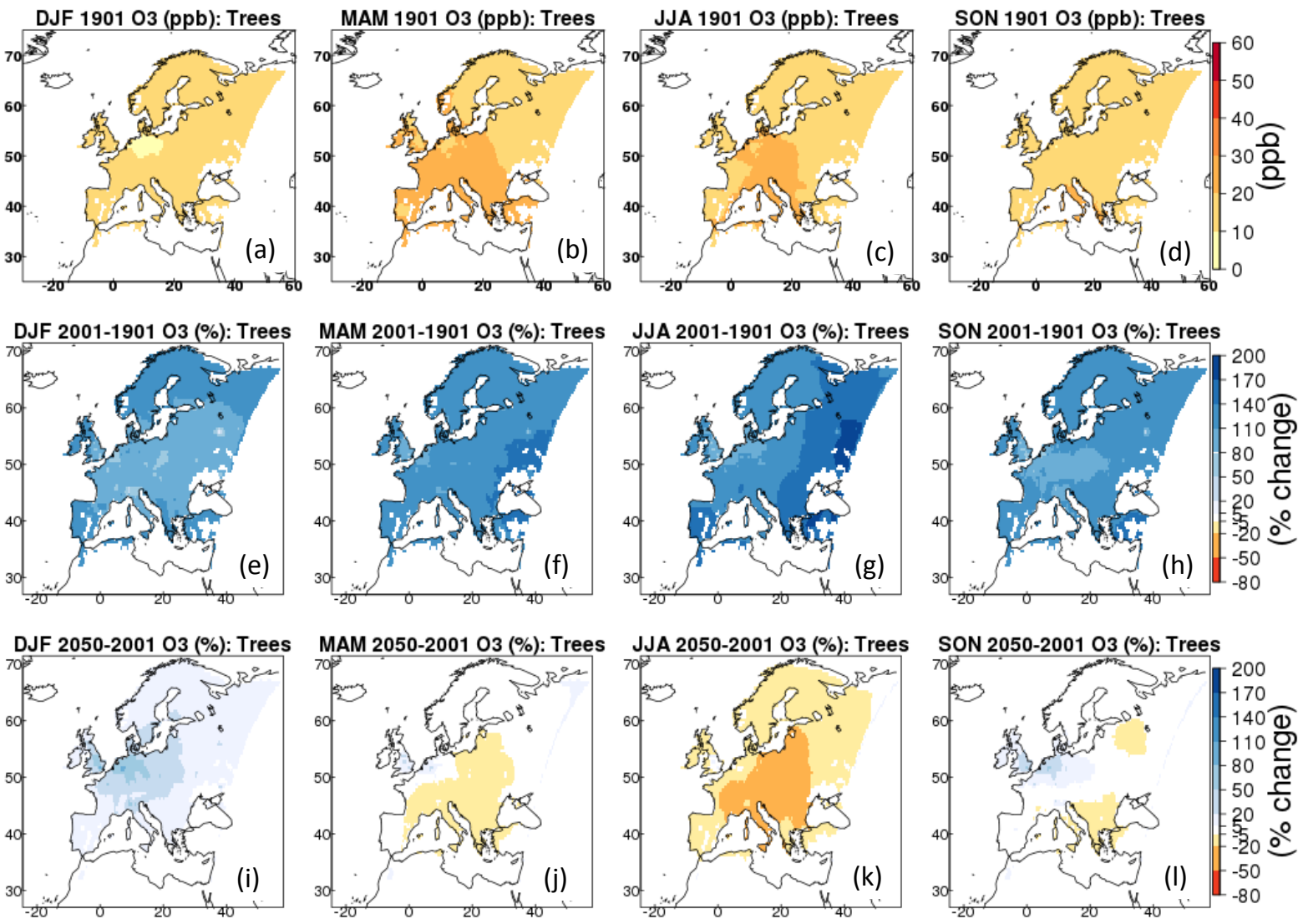

Figure S7. (a-d) 1901 seasonal mean (DJF, MAM, JJA, SON) O 3 concentration (ppb) from EMEP for woody (tree and shrub) PFTs;
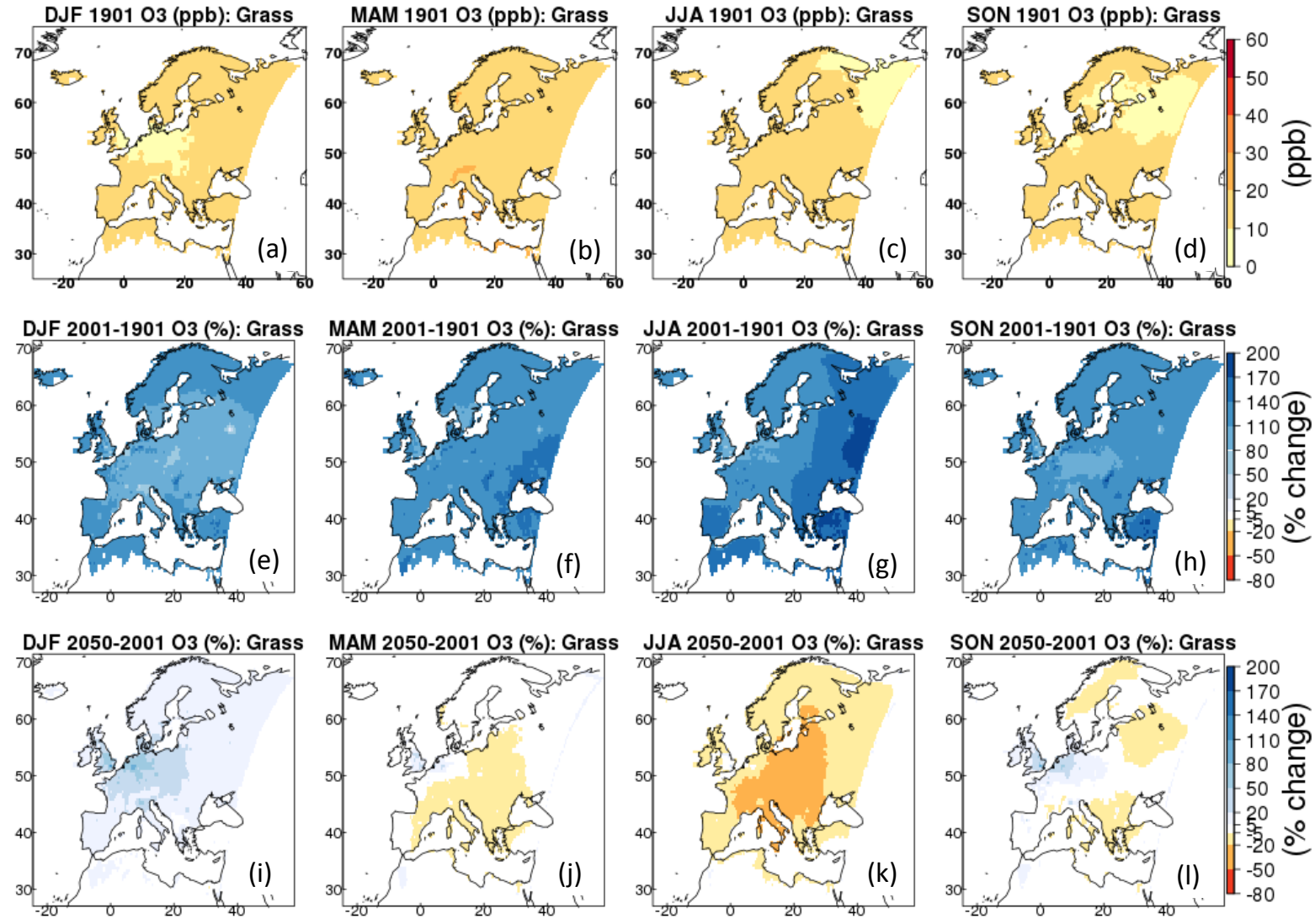

Figure S8. (a-d) 1901 seasonal mean (DJF, MAM, JJA, SON) $\mathrm{O}_{3}$ concentration (ppb) from EMEP for herbaceous PFTs; (e-h) change in seasonal $\mathrm{O}_{3}$ concentration (\%) from 1901 to 2001; (i-l) change in seasonal $\mathrm{O}_{3}$ concentration (\%) from 2001 to 2050. 

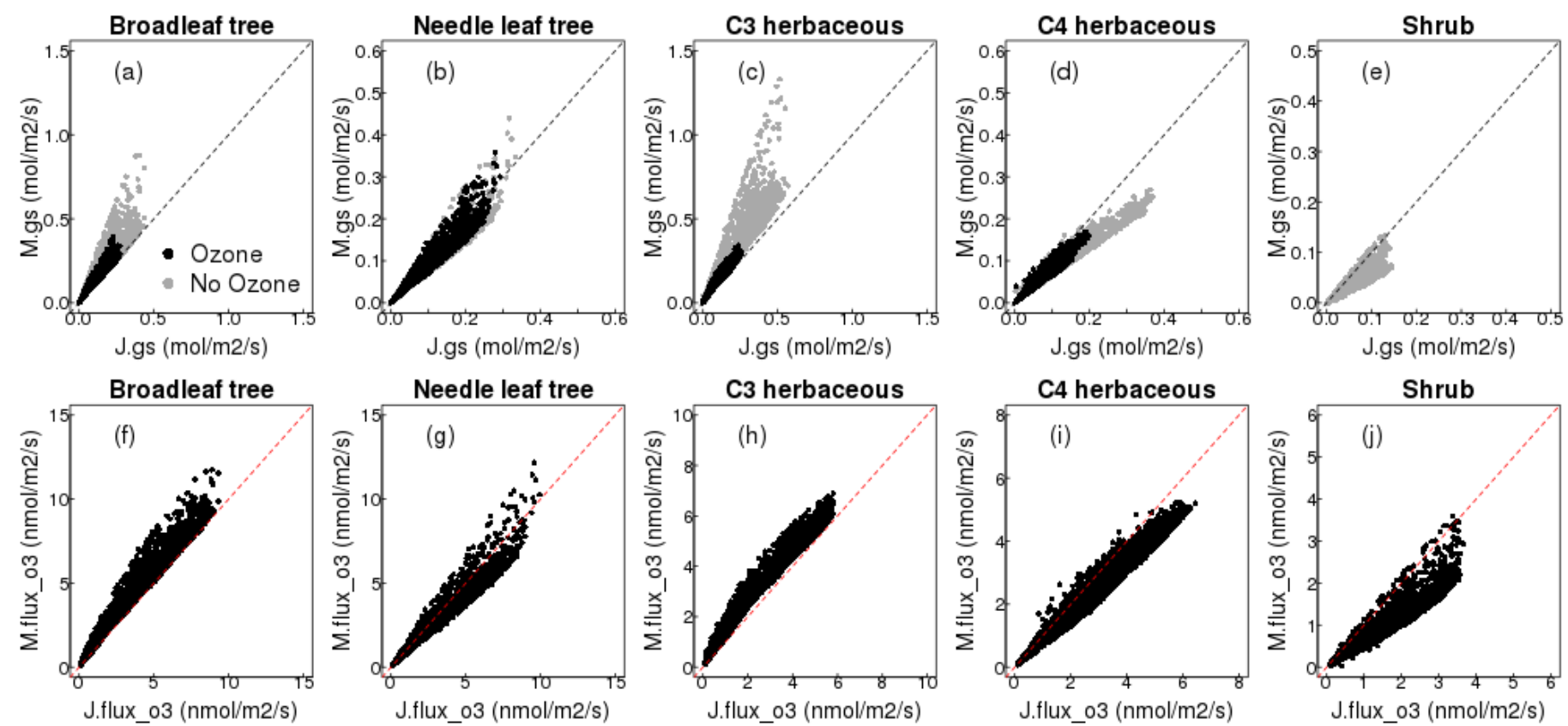

71

Figure S9. Comparison of the Medlyn et al., (2011) $g_{s}$ model (y axis) versus the Jacobs (1994) $g_{s}$ model (x axis) currently used in

72 JULES for all five JULES PFTs, for stomatal conductance ( $g s$, top row) and the flux of $\mathrm{O}_{3}$ through the stomata (flux_o3, bottom 73 row) for a dry site (high soil moisture stress). 

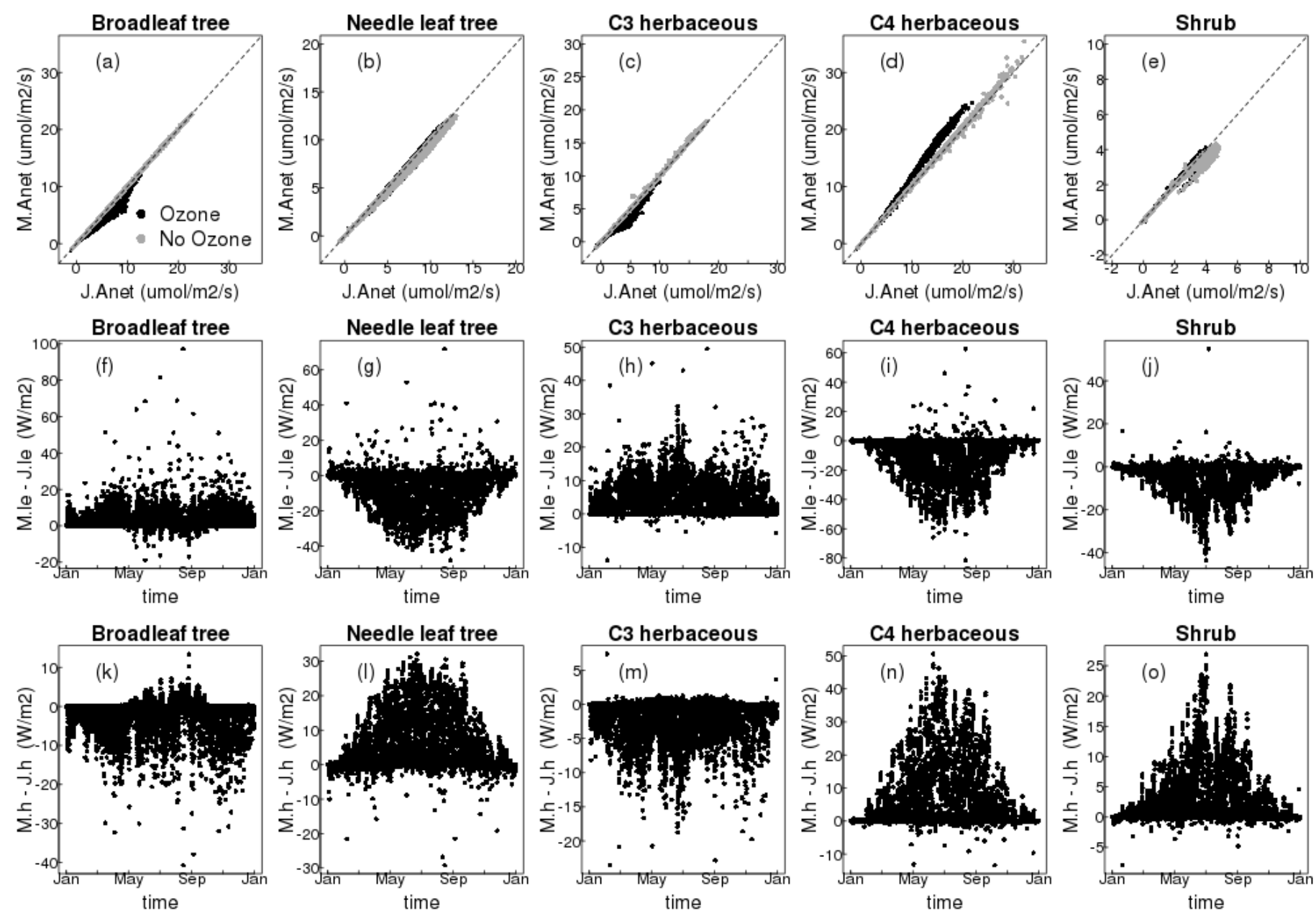

Figure S10. Comparison of the Medlyn et al., (2011) $g_{s}$ model (y axis) versus the Jacobs (1994) $g_{s}$ model (x axis) currently used in JULES for all five JULES PFTs at the wet site (low soil moisture stress), for net photosynthesis (Anet, top row). Residual plots (Medlyn - Jacobs) show the difference between models over the year for latent heat (le, middle row) and sensible heat (h, bottom row). 

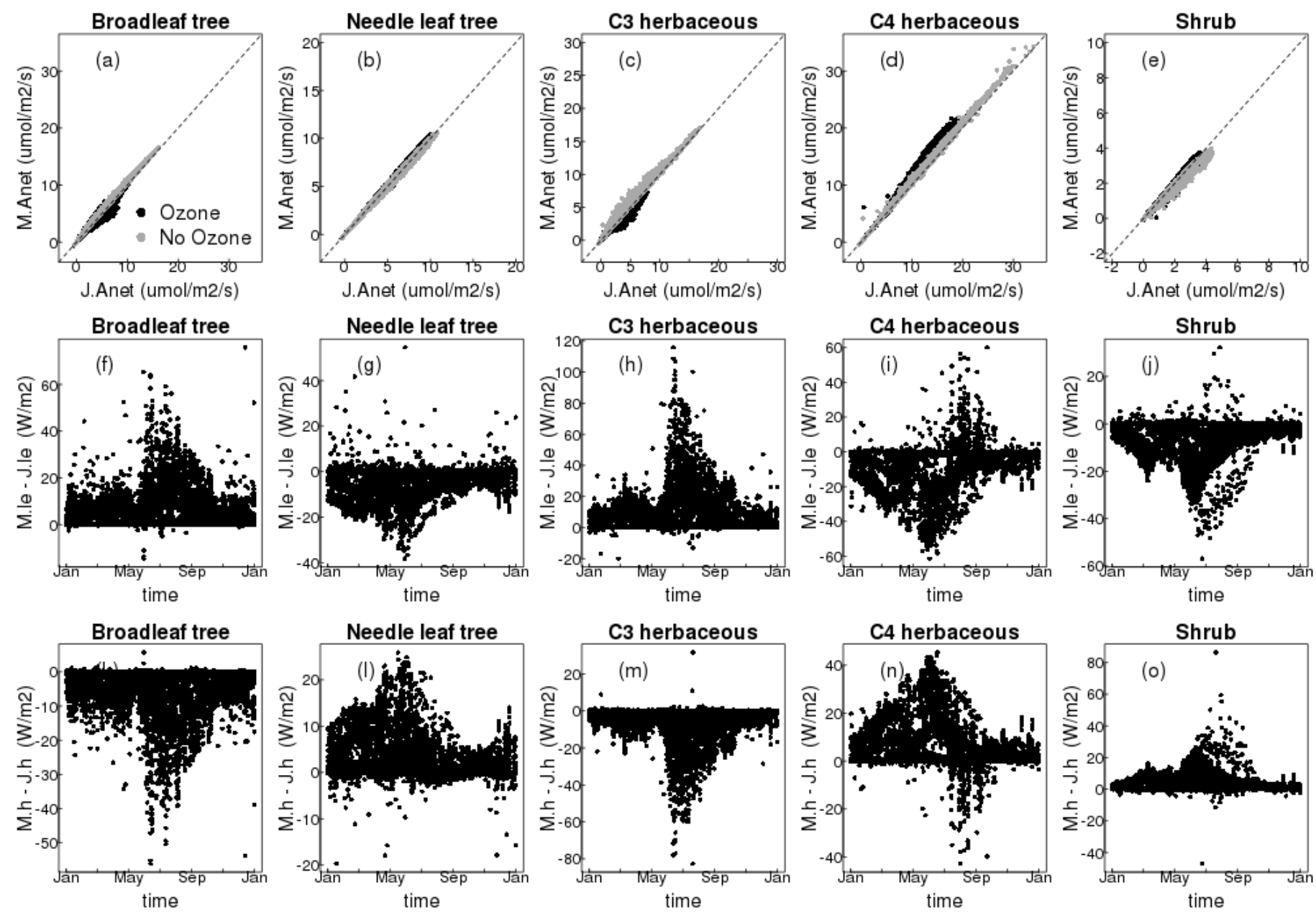

Figure S11. Comparison of the Medlyn et al., (2011) $g_{s}$ model (y axis) versus the Jacobs (1994) $g_{s}$ model (x axis) currently used in JULES for all five JULES PFTs at the dry site (high soil moisture stress), for net photosynthesis (Anet, top row). Residual plots (Medlyn - Jacobs) show the difference between models over the year for latent heat (le, middle row) and sensible heat (h, bottom row).
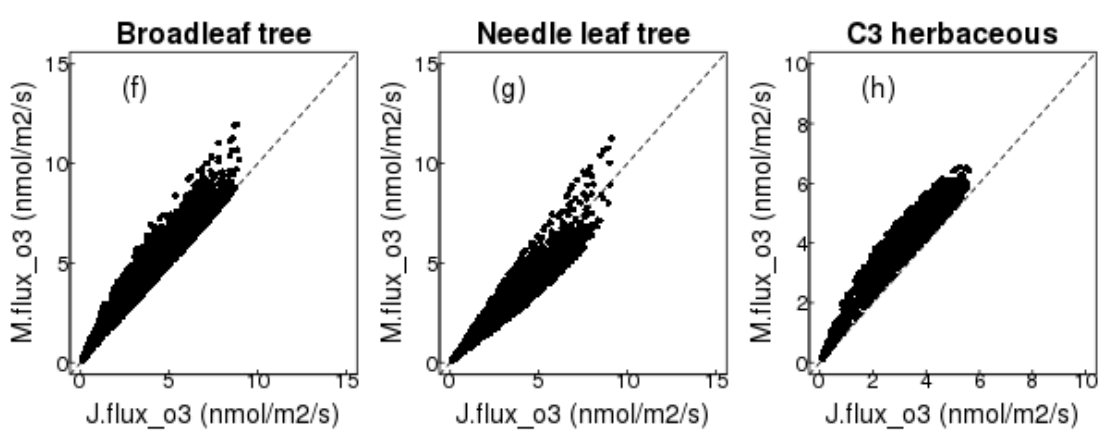

J.flux_03 (nmol/m2/s)

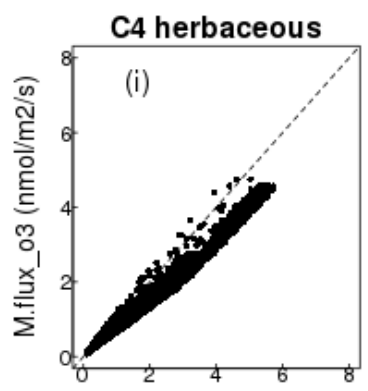

J.flux_03 (nmol/m2/s)

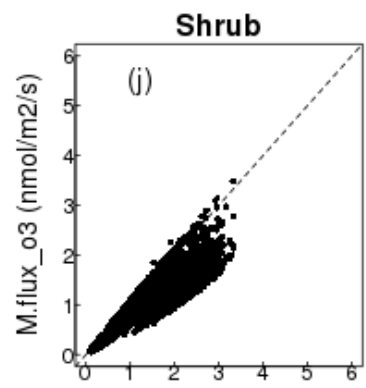

J.flux_03 (nmol/m2/s)

Figure S12. Comparison of the flux of $\mathrm{O}_{3}$ through the stomata (flux_o3) for the Medlyn et al., (2011) $g_{s}$ model (y axis) versus the Jacobs (1994) $g_{s}$ model (x axis) currently used in JULES for all five JULES PFTs for the wet site (low soil moisture stress). 
Latent Heat (W/m2) Sensible Heat (W/m2) Latent Heat $(W / m 2)$ Sensible Heat $(W / m 2)$
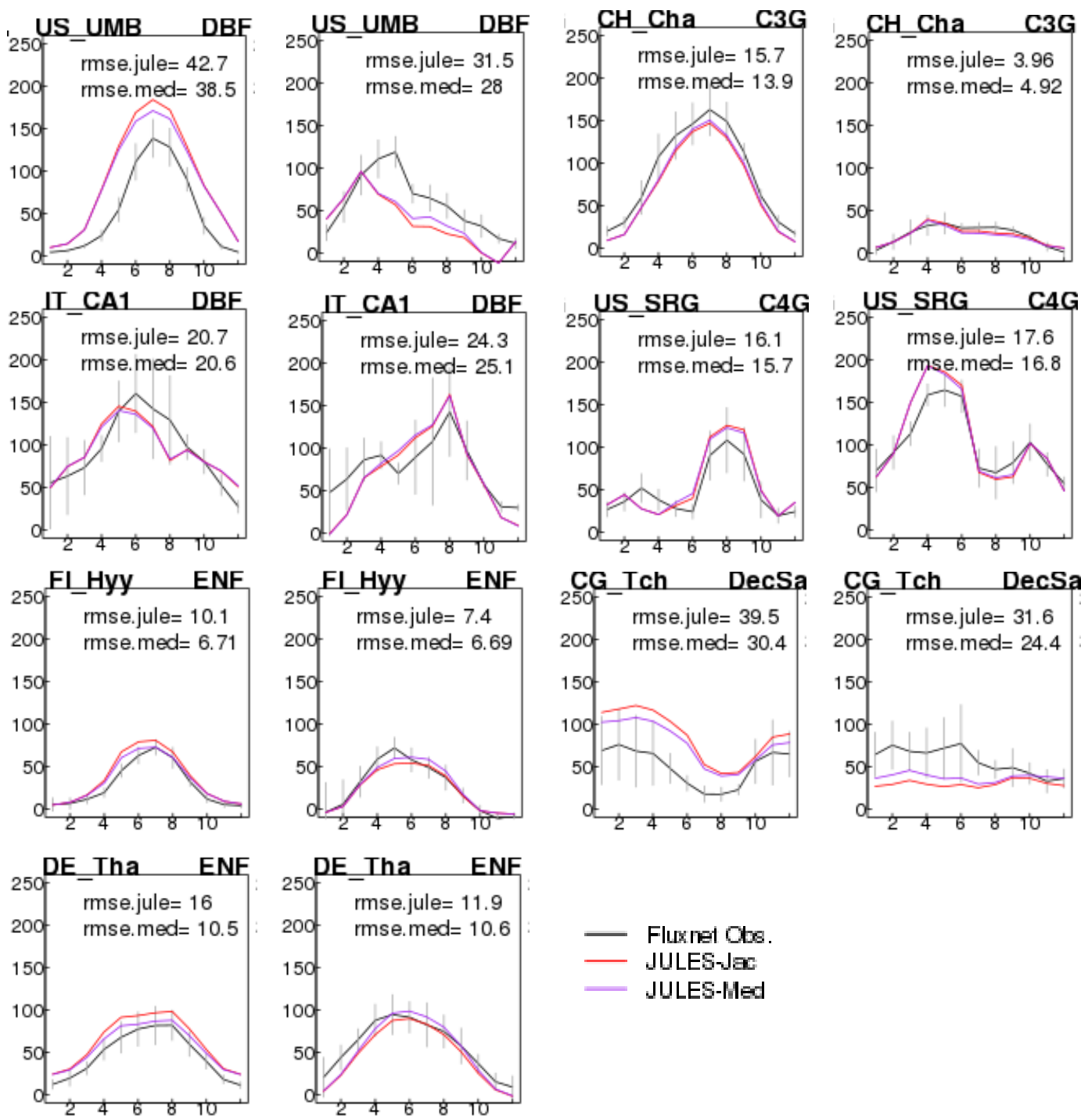

Fig. S13 Monthly mean fluxes of latent and sensible heat. Observations \pm standard deviation from FLUXNET2015 sites are shown as black line with grey vertical bars, JULES with the JAC $g_{s}$ model is shown in red and JULES with the MED $g_{s}$ model are shown in purple. Also shown are the root mean squared error (rmse) for each simulation. 

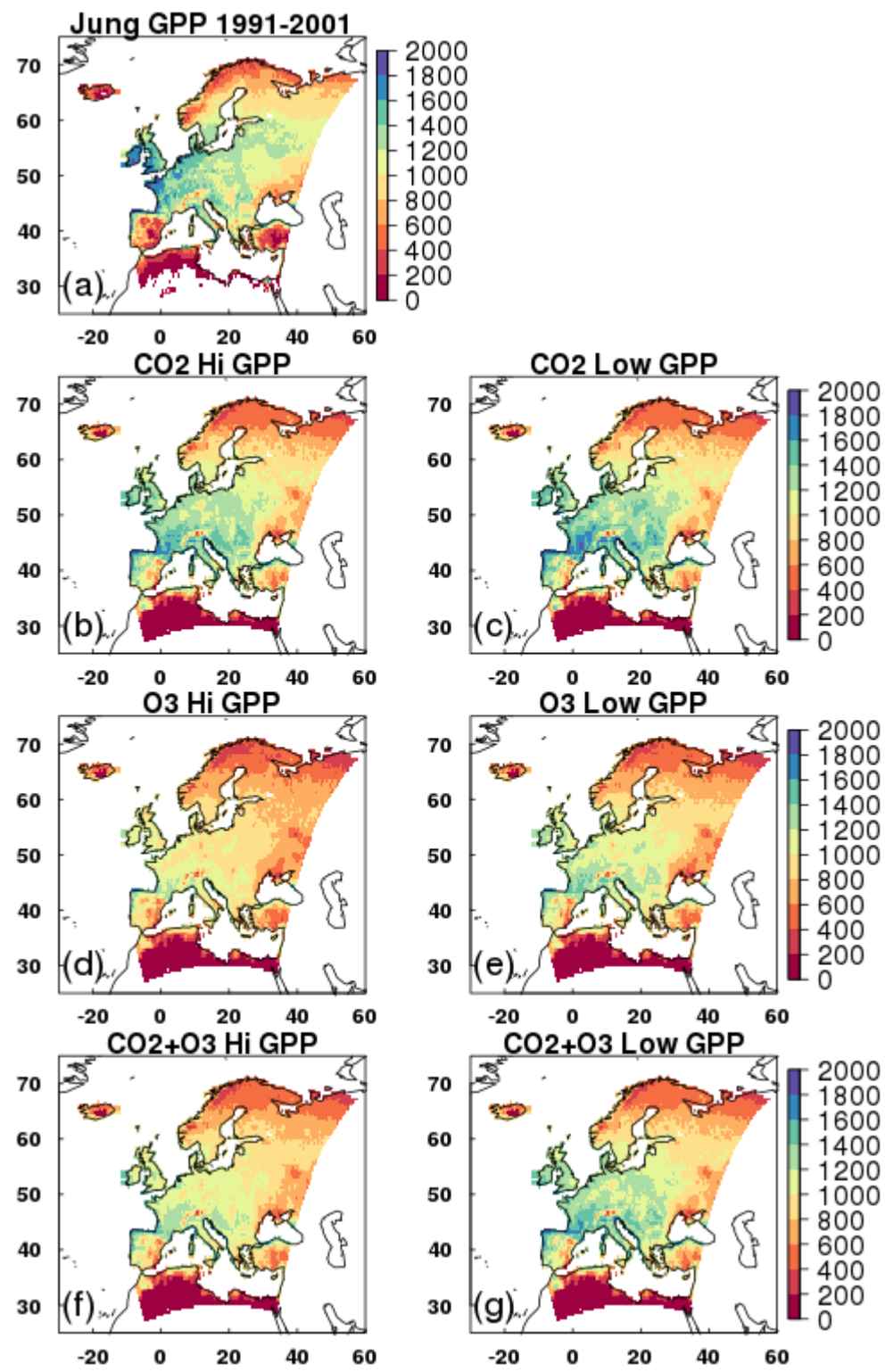

Figure S14. Mean GPP ( $\mathrm{g} \mathrm{C} \mathrm{m}^{2} \mathrm{yr}^{-1}$ ) from 1991 to 2001 for a) the observations based globally extrapolated Flux Network model tree ensemble (MTE) (Jung et al., 2011); b, c) model simulations with transient $\mathrm{CO}_{2}$ and fixed $\mathrm{O}_{3}$; d, e)model simulations with fixed $\mathrm{CO}_{2}$ and transient $\mathrm{O}_{3}$, and $\mathrm{f}, \mathrm{g}$ ) our model simulations with transient $\mathrm{CO}_{2}$ and transient $\mathrm{O}_{3}$. All model simulations show GPP for high and low plant $\mathrm{O}_{3}$ sensitivity respectively. 


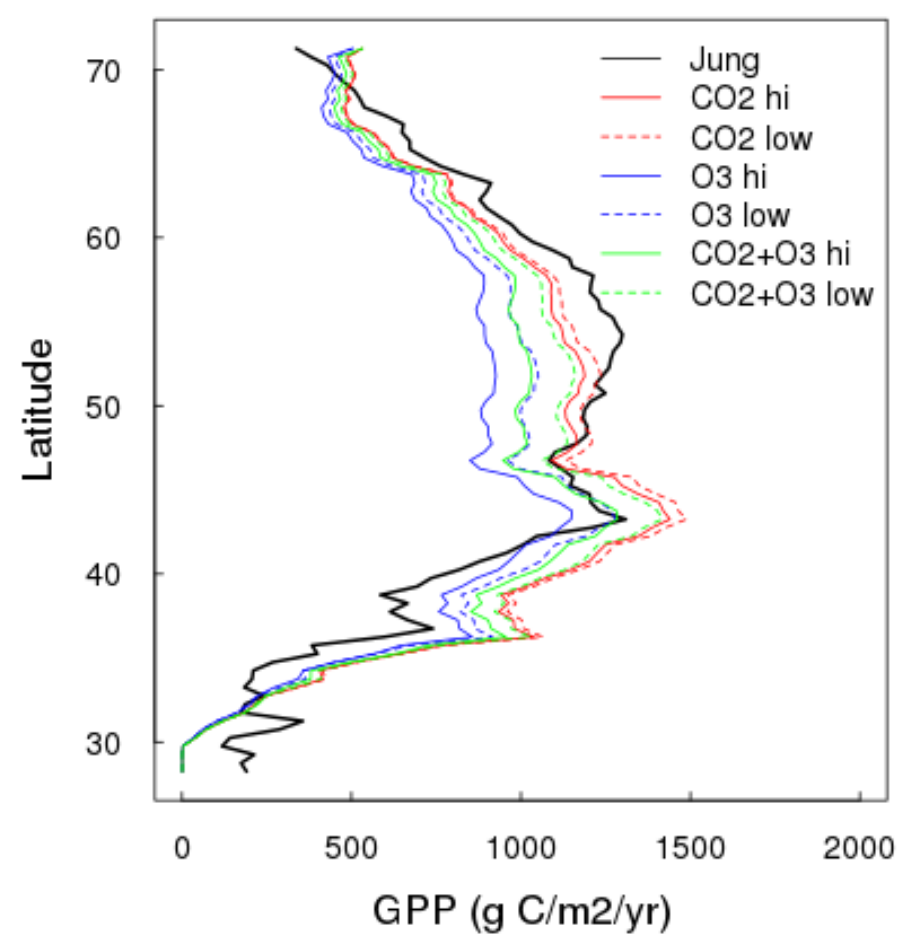

Figure S15. Zonal mean GPP from 1991 to 2001 for FLUXNET-MTE (Jung) and all JULES scenario simulations with both high (solid lines) and low (dashed lines) plant $\mathrm{O}_{3}$ sensitivity.

Mediterranean

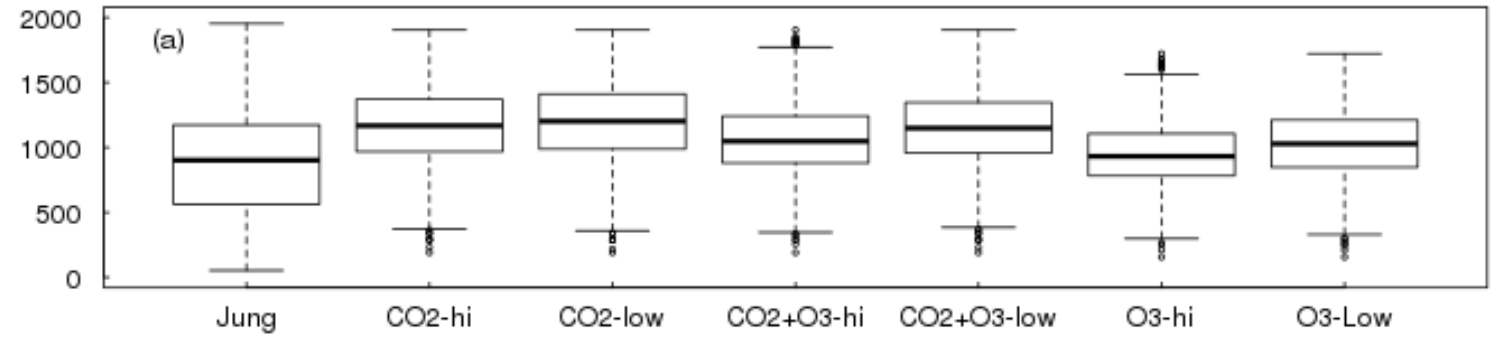

Temperate

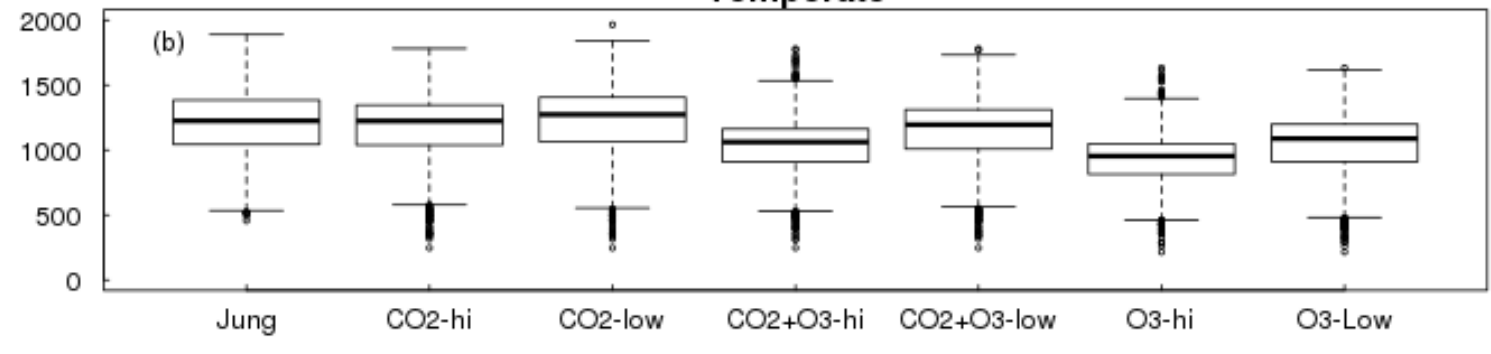

Boreal

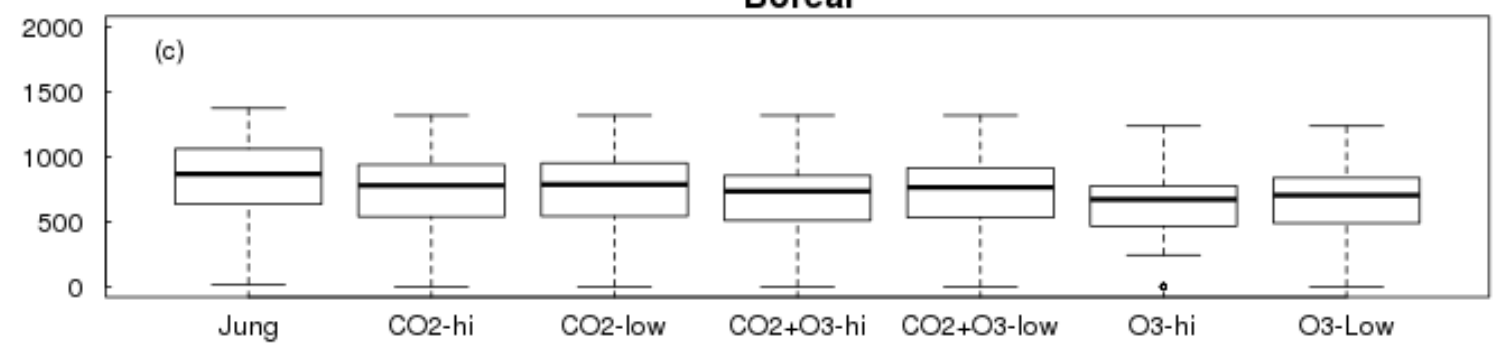

Figure S16. Mean GPP from 1991 to 2001 shown by region, comparing MTE (Jung) and all JULES scenario simulations with both high and low plant $\mathrm{O}_{3}$ sensitivity. 
Future run, constant climate (1901 - 2001)

Hi Sensitivity

\begin{tabular}{|c|c|c|c|c|c|c|}
\hline & 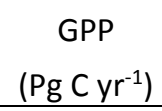 & $\begin{array}{c}\text { NPP } \\
\left(\mathrm{Pg} \mathrm{C} \mathrm{yr}^{-1}\right)\end{array}$ & $\begin{array}{c}g_{s} \\
(\mathrm{~m} / \mathrm{s}) \\
\end{array}$ & $\begin{array}{l}\text { Veg C } \\
(\mathrm{Pg} \mathrm{C}) \\
\end{array}$ & $\begin{array}{l}\text { Soil C } \\
\text { (Pg C) }\end{array}$ & $\begin{array}{l}\text { Land C } \\
(\mathrm{PgC}) \\
\end{array}$ \\
\hline Value in 1901: & 9.05 & 4.46 & 0.03228 & 41.1 & 125.8 & 167 \\
\hline \multicolumn{7}{|c|}{$\begin{array}{l}\text { Absolute diff. (2001 - } \\
\text { 1901): }\end{array}$} \\
\hline $\mathrm{O}_{3}$ & -0.81 & -0.47 & 0.00 & -0.02 & -9.09 & -9.21 \\
\hline $\mathrm{CO}_{2}$ & 1.16 & 0.76 & 0.00 & 2.82 & 1.52 & 4.24 \\
\hline $\mathrm{CO}_{2}+\mathrm{O}_{3}$ & 0.13 & 0.12 & 0.00 & 2.37 & -5.55 & -3.28 \\
\hline Relative diff. (\%) & $(\%)$ & $(\%)$ & $(\%)$ & $(\%)$ & $(\%)$ & $(\%)$ \\
\hline $\mathrm{O}_{3}$ & -8.95 & -10.54 & -8.55 & -0.05 & -7.23 & -5.51 \\
\hline $\mathrm{CO}_{2}$ & 12.82 & 17.04 & -6.07 & 6.86 & 1.21 & 2.54 \\
\hline \multirow[t]{3}{*}{$\mathrm{CO}_{2}+\mathrm{O}_{3}$} & 1.44 & 2.69 & -13.66 & 5.77 & -4.41 & -1.96 \\
\hline & \multicolumn{6}{|c|}{ Lower Sensitivity } \\
\hline & $\begin{array}{c}\text { GPP } \\
\left({\left.\mathrm{Pg} \mathrm{C} y r^{-1}\right)}^{0}\right.\end{array}$ & $\begin{array}{c}\text { NPP } \\
\left(\mathrm{PgC} \mathrm{yr}^{-1}\right)\end{array}$ & $\begin{array}{c}g_{s} \\
(\mathrm{~m} / \mathrm{s})\end{array}$ & $\begin{array}{l}\text { Veg C } \\
(\mathrm{Pg} \mathrm{C})\end{array}$ & $\begin{array}{l}\text { Soil C } \\
\text { (Pg C) }\end{array}$ & $\begin{array}{l}\text { Land C } \\
(\mathrm{Pg} \mathrm{C})\end{array}$ \\
\hline Value in 1901: & 9.34 & 4.65 & 0.03319 & 41.1 & 126.4 & 167.5 \\
\hline \multicolumn{7}{|c|}{$\begin{array}{l}\text { Absolute diff. (2001 - } \\
\text { 1901): }\end{array}$} \\
\hline $\mathrm{O}_{3}$ & -0.30 & -0.21 & 0.00 & -0.21 & -3.38 & -3.59 \\
\hline $\mathrm{CO}_{2}$ & 1.15 & 0.74 & 0.00 & 2.73 & 3.70 & 6.43 \\
\hline $\mathrm{CO}_{2}+\mathrm{O}_{3}$ & 0.65 & 0.43 & 0.00 & 2.21 & 0.29 & 2.50 \\
\hline Relative diff. (\%) & $(\%)$ & $(\%)$ & $(\%)$ & $(\%)$ & $(\%)$ & $(\%)$ \\
\hline $\mathrm{O}_{3}$ & -3.21 & -4.52 & -3.31 & -0.51 & -2.67 & -2.14 \\
\hline $\mathrm{CO}_{2}$ & 12.31 & 15.91 & -6.39 & 6.64 & 2.93 & 3.84 \\
\hline $\mathrm{CO}_{2}+\mathrm{O}_{3}$ & 6.96 & 9.25 & -9.88 & 5.38 & 0.23 & 1.49 \\
\hline
\end{tabular}

Table S3. Simulated changes in the European land carbon cycle due to changing $\mathrm{O}_{3}$ and $\mathrm{CO}_{2}$ concentrations. Shown are changes in total carbon stocks (Land C), split into vegetation (Veg C) and soil (Soil C) carbon, and gross primary productivity (GPP), net 
$\mathrm{Hi}: 03$

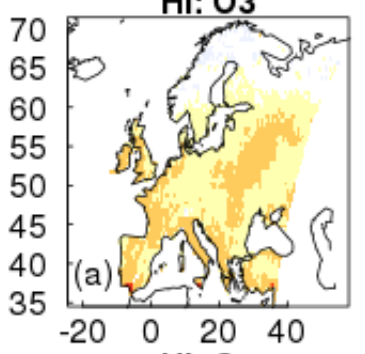

$\mathrm{Hi}: \mathrm{O3}$

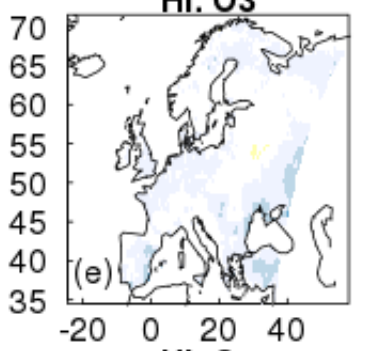

$\mathrm{Hi}: \mathrm{O3}^{2}$

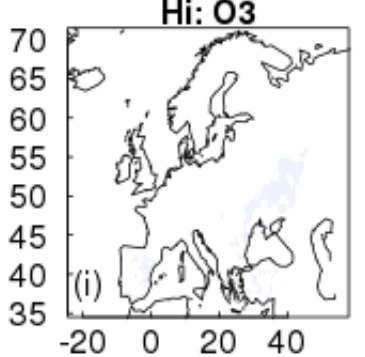

$\mathrm{Hi}: \mathrm{CO} 2$

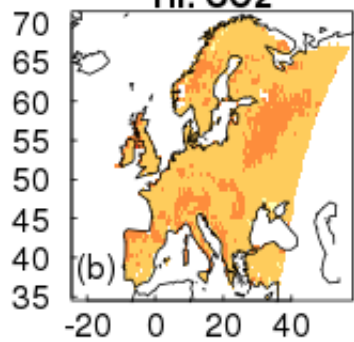

$\mathrm{Hi}: \mathrm{CO} 2$

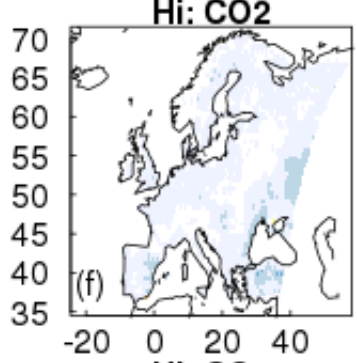

$\mathrm{Hi}: \mathrm{CO} 2$

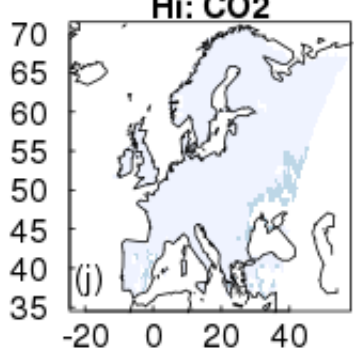

$\mathrm{Hi}: \mathrm{CO}+\mathrm{O} 3$

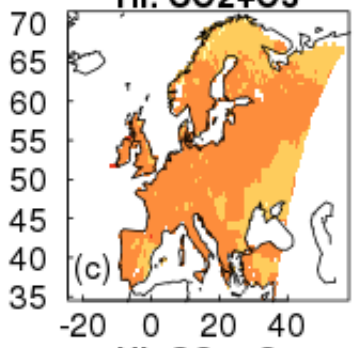

$\mathrm{Hi}: \mathrm{CO} 2+\mathrm{O} 3$

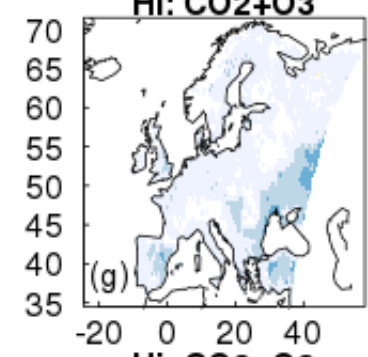

$\mathrm{Hi}: \mathrm{CO} 2+\mathrm{O} 3$

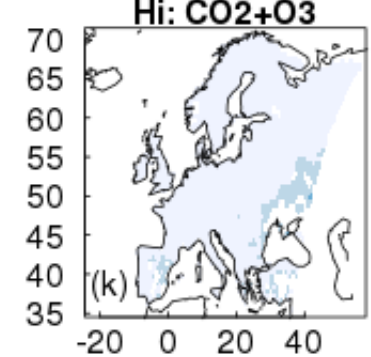

$\mathrm{Hi}: \mathrm{CO} 2(03)$
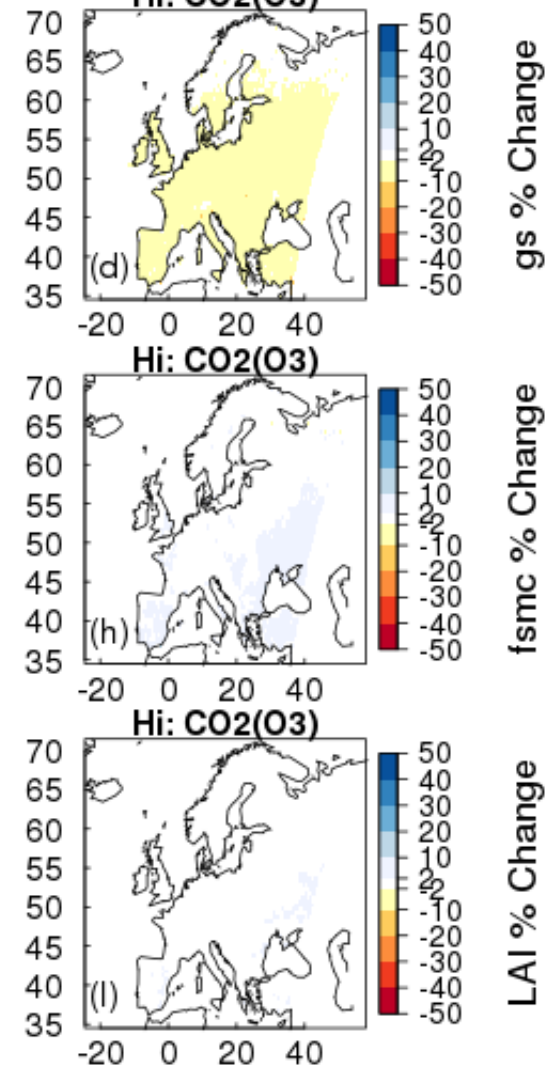

147

148

149

150

151

Figure S17. Simulated percentage change in stomatal conductance (gs) a-c), soil moisture availability factor (fsmc) d-e) and leaf area index $(L A I)$ g-i) due to $\mathrm{O}_{3}$ effects at fixed pre-industrial atmospheric $\mathrm{CO}_{2}$ concentration $(\mathrm{O} 3), \mathrm{CO}_{2}$ effects at fixed pre-industrial $\mathrm{O}_{3}$ concentration $(\mathrm{CO} 2)$, and effects of $\mathrm{CO}_{2}$ and $\mathrm{O}_{3}$ changing simultaneously $(\mathrm{CO} 2+\mathrm{O} 3)$. Changes are shown for the periods 1901 to 2050 for the higher plant $\mathrm{O}_{3}$ sensitivity. 

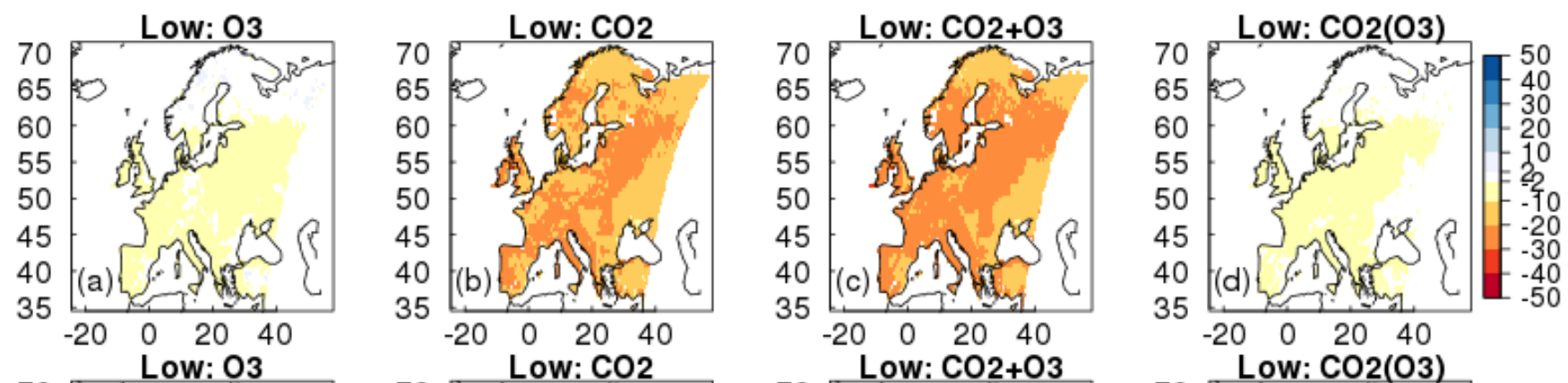

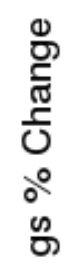
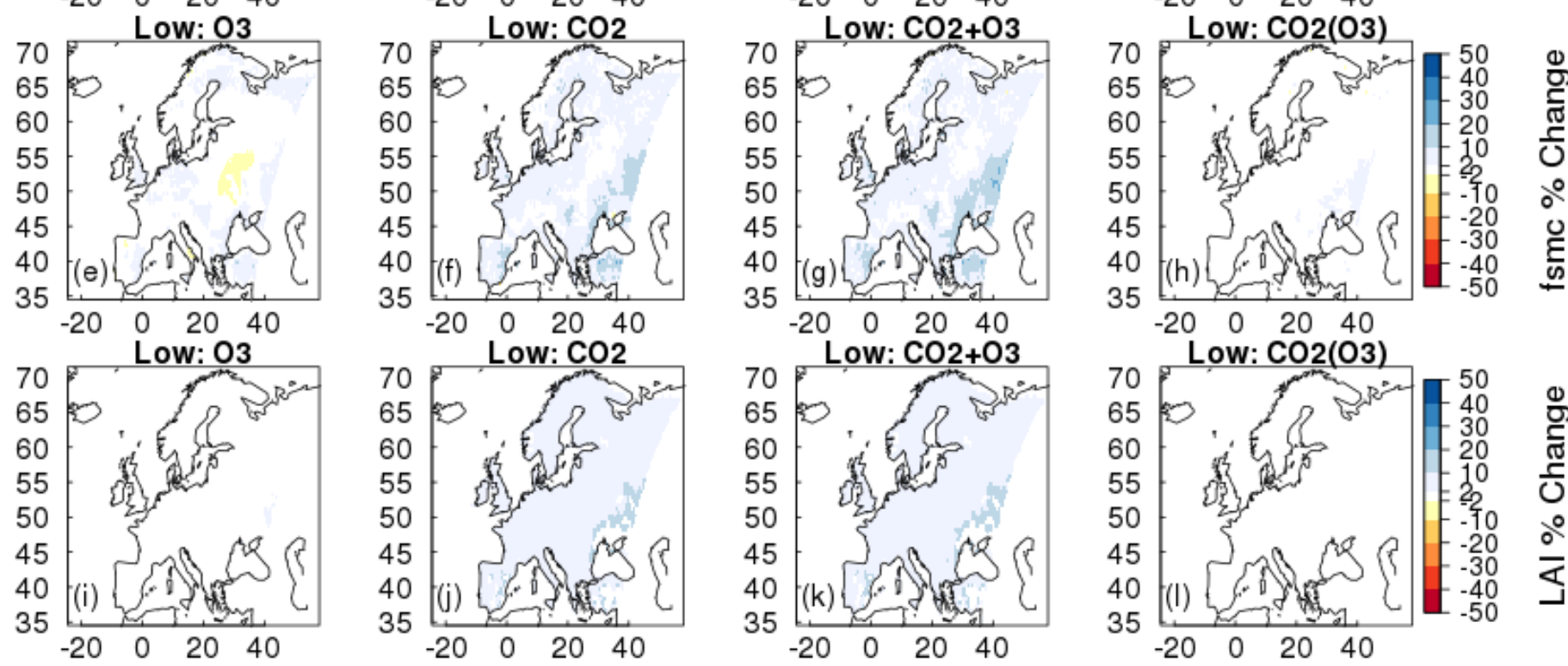

Figure S18. Simulated percentage change in stomatal conductance (gs) a-c), soil moisture availability factor (fsmc) d-e) and leaf area index $(L A I)$ g-i) due to $\mathrm{O}_{3}$ effects at fixed pre-industrial atmospheric $\mathrm{CO}_{2}$ concentration $(\mathrm{O} 3), \mathrm{CO}_{2}$ effects at fixed pre-industrial $\mathrm{O}_{3}$ concentration $(\mathrm{CO} 2)$, and effects of $\mathrm{CO}_{2}$ and $\mathrm{O}_{3}$ changing simultaneously $(\mathrm{CO} 2+\mathrm{O} 3)$. Changes are shown for the periods 1901 to 2050 for the lower plant $\mathrm{O}_{3}$ sensitivity. 
Future run, constant climate (2001 - 2050)

Hi Sensitivity

\begin{tabular}{|c|c|c|c|c|c|c|}
\hline & $\begin{array}{c}\text { GPP } \\
\left(\mathrm{PgC} \mathrm{yr}^{-1}\right)\end{array}$ & $\begin{array}{c}\text { NPP } \\
\left(\mathrm{PgC} C \mathrm{yr}^{-1}\right)\end{array}$ & $\begin{array}{c}g_{s} \\
(\mathrm{~m} / \mathrm{s})\end{array}$ & $\begin{array}{l}\text { Veg C } \\
(\mathrm{Pg} \mathrm{C})\end{array}$ & $\begin{array}{l}\text { Soil C } \\
\text { (Pg C) }\end{array}$ & $\begin{array}{l}\text { Land C } \\
(\mathrm{Pg} \mathrm{C})\end{array}$ \\
\hline \multicolumn{7}{|l|}{ Value in 2001: } \\
\hline $\mathrm{O}_{3}$ & 8.24 & 3.99 & 0.02952 & 41.08 & 116.71 & 157.79 \\
\hline $\mathrm{CO}_{2}$ & 10.21 & 5.22 & 0.03032 & 43.92 & 127.32 & 171.24 \\
\hline $\mathrm{CO}_{2}+\mathrm{O}_{3}$ & 9.18 & 4.58 & 0.02787 & 43.47 & 120.25 & 163.72 \\
\hline \multicolumn{7}{|c|}{$\begin{array}{l}\text { Absolute diff. (2050 - } \\
\text { 2001): }\end{array}$} \\
\hline $\mathrm{O}_{3}$ & 0.01 & 0.00 & 0.00 & -0.09 & -2.35 & -2.44 \\
\hline $\mathrm{CO}_{2}$ & 1.42 & 0.95 & 0.00 & 5.25 & 7.73 & 12.98 \\
\hline $\mathrm{CO}_{2}+\mathrm{O}_{3}$ & 1.66 & 1.07 & 0.00 & 5.11 & 6.00 & 11.11 \\
\hline Relative diff. (\%) & $(\%)$ & $(\%)$ & $(\%)$ & $(\%)$ & $(\%)$ & $(\%)$ \\
\hline $\mathrm{O}_{3}$ & 0.12 & 0.00 & 0.00 & -0.22 & -2.01 & -1.55 \\
\hline $\mathrm{CO}_{2}$ & 13.91 & 18.20 & -13.89 & 11.95 & 6.07 & 7.58 \\
\hline \multirow[t]{3}{*}{$\mathrm{CO}_{2}+\mathrm{O}_{3}$} & 18.08 & 23.36 & -11.37 & 11.76 & 4.99 & 6.79 \\
\hline & \multicolumn{6}{|c|}{ Lower Sensitivity } \\
\hline & $\begin{array}{c}\text { GPP } \\
\left({\left.\mathrm{Pg} \mathrm{C} y r^{-1}\right)}\right.\end{array}$ & $\begin{array}{c}\text { NPP } \\
\left(\mathrm{Pg} C \mathrm{yr}^{-1}\right) \\
\end{array}$ & $\begin{array}{c}g_{s} \\
(\mathrm{~m} / \mathrm{s})\end{array}$ & $\begin{array}{l}\text { Veg C } \\
(\mathrm{Pg} \mathrm{C})\end{array}$ & $\begin{array}{l}\text { Soil C } \\
(\mathrm{Pg} \mathrm{C})\end{array}$ & $\begin{array}{l}\text { Land C } \\
(\mathrm{Pg} \mathrm{C})\end{array}$ \\
\hline \multicolumn{7}{|l|}{ Value in 2001: } \\
\hline $\mathrm{O}_{3}$ & 9.04 & 4.44 & 0.03 & 40.89 & 123.02 & 163.91 \\
\hline $\mathrm{CO}_{2}$ & 10.49 & 5.39 & 0.03 & 43.83 & 130.1 & 173.93 \\
\hline $\mathrm{CO}_{2}+\mathrm{O}_{3}$ & 9.99 & 5.08 & 0.02991 & 43.31 & 126.69 & 170 \\
\hline \multicolumn{7}{|c|}{$\begin{array}{l}\text { Absolute diff. (2050 - } \\
\text { 2001): }\end{array}$} \\
\hline $\mathrm{O}_{3}$ & 0.02 & -0.06 & 0.00 & -0.13 & -0.94 & -1.07 \\
\hline $\mathrm{CO}_{2}$ & 1.35 & 0.92 & 0.00 & 5.25 & 7.89 & 13.14 \\
\hline $\mathrm{CO}_{2}+\mathrm{O}_{3}$ & 1.50 & 1.00 & 0.00 & 5.11 & 7.25 & 12.35 \\
\hline Relative diff. (\%) & (\%) & $(\%)$ & $(\%)$ & (\%) & $(\%)$ & $(\%)$ \\
\hline $\mathrm{O}_{3}$ & 0.22 & -1.35 & -0.72 & -0.32 & -0.76 & -0.65 \\
\hline $\mathrm{CO}_{2}$ & 12.87 & 17.07 & -14.64 & 11.98 & 6.06 & 7.55 \\
\hline $\mathrm{CO}_{2}+\mathrm{O}_{3}$ & 15.02 & 19.69 & -13.37 & 11.80 & 5.72 & 7.26 \\
\hline
\end{tabular}

Table S4. Simulated changes in the European land carbon cycle due to changing $\mathrm{O}_{3}$ and $\mathrm{CO}_{2}$ concentrations. Shown are changes in total carbon stocks (Land C), split into vegetation (Veg C) and soil (Soil C) carbon, and gross primary productivity (GPP), net primary productivity (NPP) and conductance $\left(g_{s}\right)$, between 2001 and 2050. 


\begin{tabular}{|c|c|c|c|c|c|c|}
\hline & $\begin{array}{c}\text { GPP } \\
\left(\mathrm{Pg} \mathrm{C} \mathrm{yr}^{-1}\right)\end{array}$ & $\begin{array}{c}\text { NPP } \\
\left(\mathrm{Pg} \mathrm{C} \mathrm{yr}^{-1}\right)\end{array}$ & $\begin{array}{c}g_{s} \\
(\mathrm{~m} / \mathrm{s})\end{array}$ & $\begin{array}{l}\operatorname{Veg} C \\
(P g C)\end{array}$ & $\begin{array}{l}\text { Soil C } \\
(\mathrm{Pg} \mathrm{C})\end{array}$ & $\begin{array}{l}\text { Land C } \\
(\mathrm{Pg} \mathrm{C})\end{array}$ \\
\hline Value in 1901: & 9.05 & 4.46 & 0.03228 & 41.1 & 125.8 & 167 \\
\hline \multicolumn{7}{|c|}{$\begin{array}{l}\text { Absolute diff. (2050 - } \\
\text { 1901): }\end{array}$} \\
\hline $\mathrm{O}_{3}$ & -0.80 & -0.47 & 0.00 & -0.11 & -11.44 & -11.65 \\
\hline $\mathrm{CO}_{2}$ & 2.58 & 1.71 & -0.01 & 8.07 & 9.25 & 17.22 \\
\hline $\mathrm{CO}_{2}+\mathrm{O}_{3}$ & 1.79 & 1.19 & -0.01 & 7.48 & 0.45 & 7.83 \\
\hline Relative diff. (\%) & $(\%)$ & $(\%)$ & $(\%)$ & $(\%)$ & $(\%)$ & $(\%)$ \\
\hline $\mathrm{O}_{3}$ & -8.84 & -10.54 & -8.55 & -0.27 & -9.09 & -6.98 \\
\hline $\mathrm{CO}_{2}$ & 28.51 & 38.34 & -19.11 & 19.64 & 7.35 & 10.31 \\
\hline \multirow[t]{3}{*}{$\mathrm{CO}_{2}+\mathrm{O}_{3}$} & 19.78 & 26.68 & -23.48 & 18.20 & 0.36 & 4.69 \\
\hline & \multicolumn{6}{|c|}{ Lower Sensitivity } \\
\hline & $\begin{array}{c}\text { GPP } \\
\left(\mathrm{Pg} \mathrm{C} \mathrm{yr}^{-1}\right)\end{array}$ & $\begin{array}{c}\text { NPP } \\
\left(\mathrm{Pg} \mathrm{C} \mathrm{yr}^{-1}\right)\end{array}$ & $\begin{array}{c}g_{s} \\
(\mathrm{~m} / \mathrm{s})\end{array}$ & $\begin{array}{l}\operatorname{Veg} C \\
(\mathrm{Pg} \mathrm{C})\end{array}$ & $\begin{array}{l}\text { Soil C } \\
\text { (Pg C) }\end{array}$ & $\begin{array}{l}\text { Land C } \\
(\mathrm{Pg} \mathrm{C})\end{array}$ \\
\hline Value in 1901: & 9.34 & 4.65 & 0.03319 & 41.1 & 126.4 & 167.5 \\
\hline \multicolumn{7}{|c|}{$\begin{array}{l}\text { Absolute diff. (2050 - } \\
\text { 1901): }\end{array}$} \\
\hline $\mathrm{O}_{3}$ & -0.40 & -0.27 & 0.00 & -0.34 & -4.32 & -4.66 \\
\hline $\mathrm{CO}_{2}$ & 2.50 & 1.66 & -0.01 & 7.98 & 11.59 & 19.57 \\
\hline $\mathrm{CO}_{2}+\mathrm{O}_{3}$ & 2.15 & 1.43 & -0.01 & 7.32 & 7.54 & 14.85 \\
\hline Relative diff. (\%) & $(\%)$ & $(\%)$ & $(\%)$ & $(\%)$ & $(\%)$ & $(\%)$ \\
\hline $\mathrm{O}_{3}$ & -4.28 & -5.81 & -4.01 & -0.83 & -3.42 & -2.78 \\
\hline $\mathrm{CO}_{2}$ & 26.77 & 35.70 & -20.10 & 19.42 & 9.17 & 11.68 \\
\hline $\mathrm{CO}_{2}+\mathrm{O}_{3}$ & 23.02 & 30.75 & -21.93 & 17.81 & 5.97 & 8.87 \\
\hline
\end{tabular}

Table S5. Simulated changes in the European land carbon cycle due to changing $\mathrm{O}_{3}$ and $\mathrm{CO}_{2}$ concentrations. Shown are changes in total carbon stocks (Land C), split into vegetation (Veg C) and soil (Soil C) carbon, and gross primary productivity (GPP), net primary productivity (NPP) and conductance $\left(g_{s}\right)$, between 1901 and 2050. 


\begin{tabular}{|c|c|c|c|c|}
\hline & $\begin{array}{c}\text { GPP_hi } \\
\left(\mathrm{Pg} \mathrm{C} \mathrm{yr}^{-1}\right)\end{array}$ & $\begin{array}{l}\text { GPP_low } \\
\left(\mathrm{Pg} \mathrm{C} \mathrm{yr}^{-1}\right)\end{array}$ & $\begin{array}{c}\text { LandC_hi } \\
\text { (Pg C) }\end{array}$ & $\begin{array}{c}\text { LandC_low } \\
(\mathrm{Pg} \mathrm{C})\end{array}$ \\
\hline Value in 1901: & 9.05 & 9.34 & 167.00 & 167.50 \\
\hline \multicolumn{5}{|l|}{ Value in 2050: } \\
\hline $\mathrm{CO}_{2}$ & 11.63 & 11.84 & 184.22 & 187.07 \\
\hline $\mathbf{O}_{3}$ & 8.25 & 8.94 & 155.35 & 162.84 \\
\hline $\mathrm{CO}_{2}+\mathrm{O}_{3}$ & 10.84 & 11.49 & 174.83 & 182.35 \\
\hline$\dagger \%$ change due to $\mathrm{O}_{3}$ at $\mathrm{PICO}_{2}$ & -8.84 & -4.28 & -6.98 & -2.78 \\
\hline$\ddagger \%$ change due to $\mathrm{O}_{3}$ at high $\mathrm{CO}_{2}$ & -6.79 & -2.96 & -5.10 & -2.52 \\
\hline \multicolumn{5}{|l|}{++ Alleviation of $\mathrm{O}_{3}$ damage by $\mathrm{CO}_{2}$ increase } \\
\hline (\%) & 2.05 & 1.33 & 1.88 & 0.26 \\
\hline
\end{tabular}

LandC High

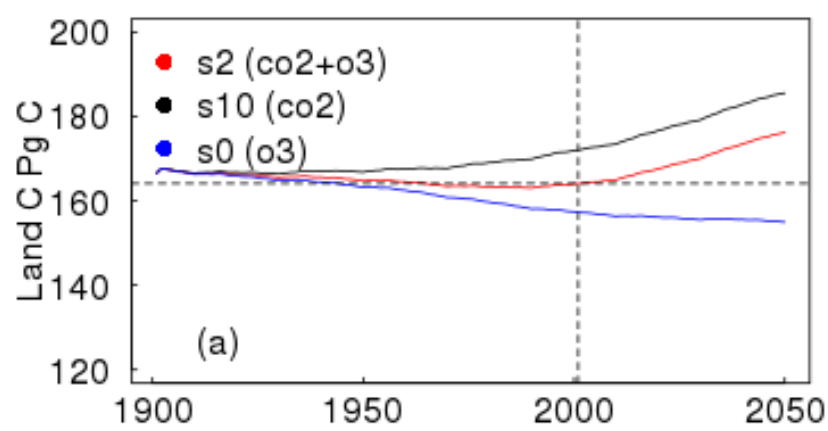

GPP High

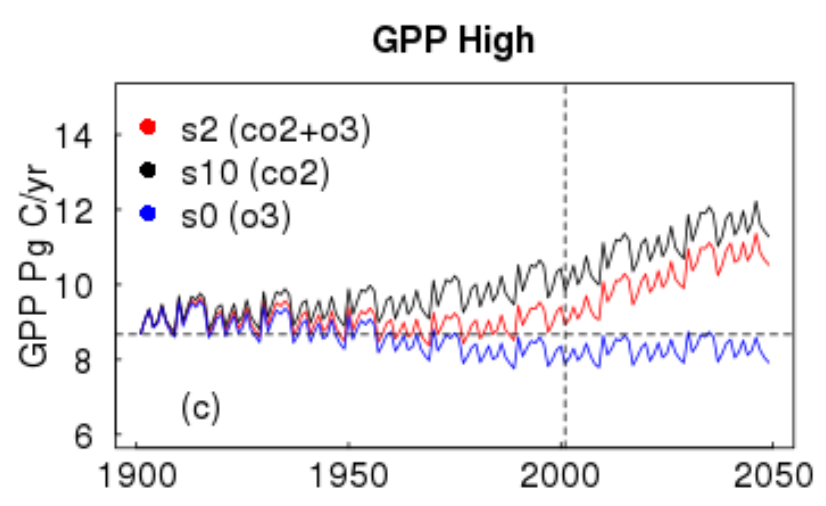

\section{LandC Low}

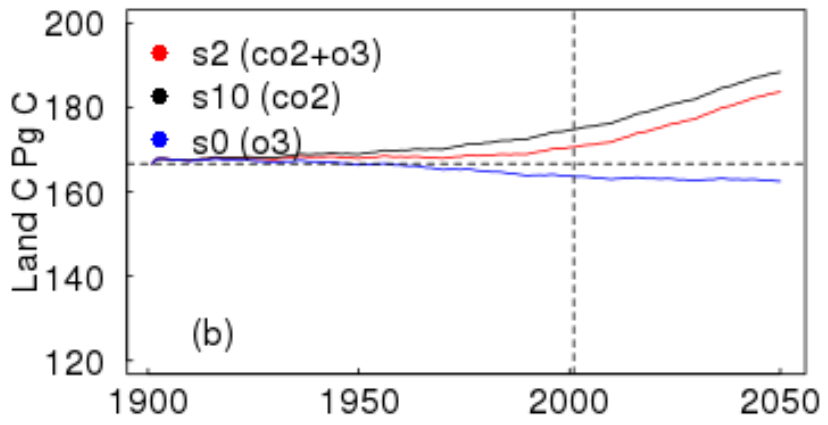

GPP Low

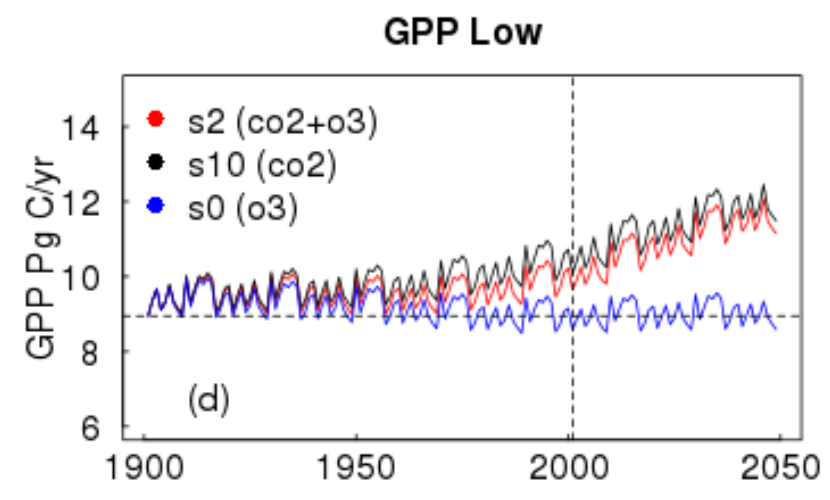

Table S6. Percentage reduction in simulated GPP and Land C by 2050 due to future $\mathrm{O}_{3}$ effects at pre-industrial (PI) $\mathrm{CO}_{2}$ concentration, and under increasing future $\mathrm{CO}_{2}$ concentration. The difference between these defines the alleviation of the $\mathrm{O}_{3}$ effect by $\mathrm{CO}_{2} . \mathbf{O}_{3}=$ Fixed $1901 \mathrm{CO}_{2}$, Varying $\mathrm{O}_{3} ; \mathbf{C O}_{2}=$ Varying $\mathrm{CO}_{2}$, Fixed $1901 \mathrm{O}_{3} ; \mathbf{C O}_{2}+\mathbf{O}_{3}=$ Varying $\mathrm{CO}_{2}$, Varying $\mathrm{O}_{3}$. Calculated as: $\dagger) \mathrm{O}_{3}$ effect with fixed pre-industrial $\mathrm{CO}_{2}: 100 *\left(\mathrm{fixCO}_{2} \mathrm{varO}_{3}\right.$ [2050] - value[1901])/value[1901], where value[1901] represents the hypothetical value at 2050 from a run with fixCO $\mathrm{CO}_{2} \mathrm{fixO}_{3}$ which is equivalent to the initial state, i.e. the value in $\left.1901 ; \vdots\right) \mathrm{O}_{3}$ effect with increasing $\mathrm{CO}_{2}: 100 *\left(\operatorname{varCO}_{2} \mathrm{varO}_{3}[2050]-\operatorname{varCO}{ }_{2} \mathrm{fixO}_{3}[2050]\right) / \mathrm{varCO}_{2} \mathrm{fixO}_{3}[2050]$; $\left.\dagger\right)$ the alleviation of $\mathrm{O}_{3}$ damage by $\mathrm{CO}_{2}$ is the difference between the two runs: $\ddagger-\uparrow$. 
Figure S19. Times series (1901 to 2050) of changes in total carbon stocks (Land C) and gross primary productivity (GPP) due to $\mathrm{O}_{3}$ effects at fixed pre-industrial atmospheric $\mathrm{CO}_{2}$ concentration $\left(\mathrm{O}_{3}\right.$, blue), $\mathrm{CO}_{2}$ effects at fixed pre-industrial $\mathrm{O}_{3}$ concentration $\left(\mathrm{CO}_{2}\right.$, black), and effects of $\mathrm{CO}_{2}$ and $\mathrm{O}_{3}$ together $\left(\mathrm{CO}_{2}+\mathrm{O}_{3}\right.$, red), for the higher and lower plant $\mathrm{O}_{3}$ sensitivity. The horizontal dashed line shows the pre-industrial value, and the vertical dashed line marks the year 2001.

\section{Acknowledgments}

This work used eddy covariance data acquired and shared by the FLUXNET community, including these networks: AmeriFlux, AfriFlux, AsiaFlux, CarboAfrica, CarboEuropeIP, CarboItaly, CarboMont, ChinaFlux, Fluxnet-Canada, GreenGrass, ICOS, KoFlux, LBA, NECC, OzFlux-TERN, TCOS-Siberia, and USCCC. The ERA-Interim reanalysis data are provided by ECMWF and processed by LSCE. The FLUXNET eddy covariance data processing and harmonization was carried out by the European Fluxes Database Cluster, AmeriFlux Management Project, and Fluxdata project of FLUXNET, with the support of CDIAC and ICOS Ecosystem Thematic Center, and the OzFlux, ChinaFlux and AsiaFlux offices. 